\title{
Instrumentation for Far-Infrared Spectroscopy
}

\author{
Peter R. Griffiths \\ Department of Chemistry \\ University of Idaho \\ Moscow, ID 83844-2343, USA
}

Telephone: $208-885-5807$

Fax: 208-885-6173

E-mail: pgriff@uidaho.edu

and

Christopher Homes

Department of Physics - Bldg. 510B, Room 1-17

Brookhaven National Laboratory

20 Pennsylvania Street

P.O. Box 5000

Upton, NY 11973-5000, USA

Telephone: Tel: (631) 344-7579

Fax: $631-344-2739$

E-mail: homes@bnl.gov 



\section{Sources for Far-Infrared Spectrometry}

Measurements of far-infrared spectra are made far more difficult than the corresponding measurement of mid- or near-infrared spectra because of the weakness of the source. The spectral energy density of a black-body at $1400 \mathrm{~K}$ is almost 5800 times weaker at $10 \mathrm{~cm}^{-1}$, and about 60 times weaker at $100 \mathrm{~cm}^{-1}$, than it is at $1000 \mathrm{~cm}^{-1}$. Since the sensitivity of any measurement of a mid- or far-infrared spectrum is directly proportional to the spectral energy density of the source, it is apparent that far-infrared sources should be as hot as possible and have an emittance of close to unity.

For measurements between about 400 and $100 \mathrm{~cm}^{-1}$, the radiant power emitted by a silicon carbide (Globar ${ }^{\mathrm{TM}}$ ) source is as high as any conventional infrared source. Although Nernst glowers can be operated at higher temperature than Globars, they become quite transparent below about $200 \mathrm{~cm}^{-1}$, so that their emissivity drops to the point that they are of little use as far-infrared sources, despite their high temperature. Below $100 \mathrm{~cm}^{-1}$, the emissivity of a Globar ${ }^{\mathrm{TM}}$ also becomes low and it is customary to use a high-pressure mercury lamp for measurements between $\sim 50 \mathrm{~cm}^{-1}$ and the onset of the microwave region of the spectrum. The reason why mercury lamps have proved to be so successful for far-infrared spectrometry is because emission from the plasma reinforces the emission from the hot quartz envelope of the lamp. However, the mercury lamp has significant flicker noise, so even if the energy is better, the signal-to-noise ratio (SNR) may not be. It should also be noted that microwave sources have been developed that emit highly monochromatic radiation at shorter wavelengths than $1 \mathrm{~mm}\left(10 \mathrm{~cm}^{-1}\right)$ but they will not be covered in this article. Because wavelengths between $1 \mathrm{~mm}$ and $100 \mu \mathrm{m}$ 
require a different measurement technology than the mid-infrared, measurements made in this region are sometimes called "submillimeter wave" spectroscopy.

The radiance of synchrotron sources in the far infrared can be as much as three orders of magnitude greater than any of the sources mentioned above [1]. From the standpoint of obtaining the highest photon flux, therefore, synchrotrons are easily the best sources of far-infrared radiation. They have two important disadvantages, however, in that "beam time" must be obtained and the experiment must be transported to the synchrotron and not vice versa. If these factors do not provide an excessive limitation, the synchrotron is certainly the ideal source of far-infrared radiation, particularly for étendue-limited applications such as reflection-absorption infrared spectroscopy of thin films.

\section{Detectors}

\subsection{Pneumatic amplifiers}

The first type of detector to be routinely used for far-infrared spectroscopy using both grating monochromators and slow- or step-scanning interferometers was the Golay pneumatic amplifier detector [2]. A schematic of one of these detectors is shown in

Figures 1 and 2. A chamber containing a gas of low thermal conductivity is sealed at one end with an infrared transparent window (A) through which radiation reaches a thin absorbing film (B). This film has a low thermal capacity, so that its temperature rises rapidly, in turn warming the gas with which it is in contact. A rise in the temperature of the gas in the chamber leads to a concomitant increase in its pressure and, therefore, a distortion of the mirror membrane (C) with which the other end of the chamber is sealed. 
A fine leak (D) connects the detection chamber with a ballasting reservoir of gas on the other side of the mirror membrane to prevent slow changes in ambient temperature from affecting the detector.

The temperature of the absorbing film follows the frequency at which the incident radiation is modulated. Visible light from a source (E) passes through a condensing lens (F) to a line grid $(\mathrm{E})$ and is focused on the flexible mirror. A meniscus lens $(\mathrm{H})$ between the line grid and the and mirror focuses the beam so that in the absence of any other deformation, an image of one part of the line grid is superimposed on another part of the same grid. If the image of a gap between lines coincides with a gap in the grid, light will be transmitted by the grid and hence the intensity of light reaching the sensor is measured.

Golay detectors are remarkably sensitive and were commonly used with slowand step-scanning interferometers and lamellar grating interferometers (vide infra). These instruments do not modulate the infrared beam at a frequency above about $0.1 \mathrm{~Hz}$, so that the beam is usually further modulated by a rotating chopper typically operating at $\sim 13 \mathrm{~Hz}$. When the older interferometers were superseded by the type of rapid-scanning interferometers incorporated in bench-top FT-IR spectrometers, the slow response speed of Golay detectors precluded their use and they were, in turn, superseded by pyroelectric bolometers.

\subsection{Pyroelectric Bolometers}

Pyroelectric bolometers incorporate as their heat-sensing element ferroelectric materials that exhibit a large spontaneous electrical polarization at temperatures below their Curie point. If the temperature of these materials is changed, the degree of 
polarization is changed. The change in polarization may be observed as an electrical signal if electrodes are placed on opposite faces of a thin slice of material to form a capacitor. When the polarization changes, the charge induced on the electrodes can either flow as a current through a relatively low external impedance or produce a voltage across the slice if the external impedance is relatively high. The detector will only give a signal when the temperature of the element changes.

The most commonly used material for pyroelectric detectors is deuterated triglycine sulfate (DTGS). The DTGS element is usually mounted so that the thermal resistance between the element and its surroundings is large, so that the thermal time constant is long. The voltage responsivity of pyroelectric detectors is approximately proportional to the reciprocal of the frequency at which the incident radiation is modulated. The frequency at which radiation of wavenumber $\tilde{v}$ is modulated by a rapidscanning interferometer is equal to $V \tilde{v} \mathrm{~Hz}$, where $V$ is the optical velocity in $\mathrm{cm} \mathrm{s}^{-1}$. For an optical velocity of $0.316 \mathrm{~cm} \mathrm{~s}^{-1}$ ( $5 \mathrm{kHz}$ laser frequency), the highest modulation frequency of far-infrared radiation (corresponding to $400 \mathrm{~cm}^{-1}$ ) is $\sim 125 \mathrm{~Hz}$, and the lowest (corresponding to $10 \mathrm{~cm}^{-1}$ ) is $3 \mathrm{~Hz}$. In principle, therefore, the performance of DTGS detectors fitted with a polyethylene window should be quite good for measurements of far-infrared spectra using a rapid-scanning interferometer. However, it should be noted that this frequency range includes electrical line frequency $(60 \mathrm{~Hz}$ in the USA) and its first harmonic, and so spikes are often seen in far-infrared spectra measured with a rapid-scanning interferometer. In addition, low-frequency building vibrations can lead to "spikes" (glitches) in the spectra at other frequencies, so that good vibration isolation is often necessary. 


\subsection{Liquid-Helium Cooled Bolometers}

Because the intensity of far-infrared radiation emitted by most sources is very low, spectra measured using pyroelectric or Golay detectors are often quite noisy. To improve the signal-to-noise ratio, it may be necessary to use a detector with much higher performance than either of these two detectors. In this case, the use of liquid helium (LHe) to cool the element is often called for.

Two types of LHe-cooled detectors have been used for the measurement of farinfrared radiation. The first are either boron-doped silicon or germanium doped with copper, gallium or antimony. When cooled with liquid ${ }^{4} \mathrm{He}$, these detectors have a low frequency cut-off of $\sim 20 \mathrm{~cm}^{-1}$. The fundamental factor limiting the sensitivity of these detectors is Johnson noise, the power of which is given by:

$$
V_{n}=\sqrt{4 k_{B} \Delta \widetilde{v} R T}
$$

where $k_{B}$ is Boltzmann's constant, $\Delta \tilde{v}$ is the bandwidth of the detector, $R$ is the resistance of the bolometer and $T$ is the operating temperature. The noise equivalent power (NEP) of these bolometers is $\sim 1.5 \times 10^{-13} \mathrm{~W} \mathrm{~Hz}^{-1 / 2}$. To minimize the effect of the mid-infrared photon flux from ambient grey bodies, LHe-cooled filters must be placed in front of these detectors. For increased sensitivity, these detectors may be operated at $1.5 \mathrm{~K}$ by pumping the helium bath through the lambda point. In this case, the NEP may be reduced as low as $8 \times 10^{-14} \mathrm{~W} \mathrm{~Hz}^{-1 / 2}$. The lambda point of liquid ${ }^{4} \mathrm{He}$ is reached by pumping on the LHe reservoir. At a pressure of $37.8 \mathrm{~mm} \mathrm{Hg}$, the liquid helium 
undergoes a superfluid transition at $2.172 \mathrm{~K}$. Continued pumping of the superfluid down to a pressure of about $1 \mathrm{~mm} \mathrm{Hg}$ lowers the temperature of the fluid to $1.27 \mathrm{~K}$.

A further increase in sensitivity can be achieved using pumped liquid ${ }^{3} \mathrm{He}$, for which the lowest operating temperature is $\sim 0.32 \mathrm{~K}$. A pumped liquid ${ }^{3} \mathrm{He}$ bolometer also has a lower bandwidth $\left(\leq 80 \mathrm{~cm}^{-1}\right.$ as opposed to $700 \mathrm{~cm}^{-1}$ for a $4.2 \mathrm{~K}$ detector). When these two factors are taken in tandem, the reduction in $V_{n}$ is an order of magnitude, and a further restriction of bandwidth can improve this figure even more. The ${ }^{3} \mathrm{He}$ bolometer has a typical NEP of $1.4 \times 10^{-15} \mathrm{~W} \mathrm{~Hz}^{-1 / 2}$, and has been used down to $1 \mathrm{~cm}^{-1}$ using a mercury arc lamp source and a lamellar grating interferometer.

The NEP of these bolometers may be compared to the typical value for a DTGS detector of $\sim 5 \times 10^{-9} \mathrm{~W} \mathrm{~Hz}^{-1 / 2}$. Thus the typical increase in sensitivity of bolometers cooled to $4.2 \mathrm{~K}$ over that of the DTGS detector at ambient temperature is more than four orders of magnitude. This improvement factor may be increased to six orders of magnitude by cooling with pumped ${ }^{3} \mathrm{He}$.

The second type of far-infrared detector is the InSb hot-electron detector. Absorption of radiation by free-carrier electrons causes their mean temperature $T_{e}$ to rise above that of the host lattice. Since electron mobility is proportional to $T_{e}^{3 / 2}$, this temperature rise can be sensed as a change in conductivity. The very low thermal mass of free electrons, with short energy relaxation times of $\sim 10^{-7} \mathrm{~s}$, leads to very fast submillimeter and millimeter wave detectors. In one modification of this type of detector, a specially-shaped $n$-type InSb crystal is coupled directly to a low-noise preamplifier without sacrificing bandwidth or introducing noise. The typical wavenumber response of these detectors is from 2 to $50 \mathrm{~cm}^{-1}$. 


\section{Interferometers}

\subsection{The Early Days}

In the late 1950s and early 1960s, two manufacturers introduced double-beam grating spectrometers for far-infrared spectrometry. These were the Perkin-Elmer (Norwalk, CT, USA) Model 301 and the Beckman (Irvine, CA, USA) IR-11. Although these instruments allowed spectra to be measured down to $33 \mathrm{~cm}^{-1}$, the spectra were noisy, especially when high resolution was required. At about the same time, John Strong, at Johns Hopkins University in Baltimore showed that it was possible to measure significantly better far-infrared spectra with an interferometer provided that the long computing times required could be tolerated. (Remember that the fast Fourier transform algorithm was not reported until 1964 and that the power of the best computers at that time was not much better than that of a small chip that played the Christmas carol "God Rest Ye Merry, Gentlemen" in Christmas cards sold in the mid 1990s.)

After Strong's work, the key developments of Michelson interferometers for farinfrared spectroscopy was carried out in the 1960s by Alastair Gebbie's group at the National Physical Laboratory (NPL) in Teddington, Middlesex, UK. Their instruments were quite small and, at least by today's standards, crude, but allowed far-infrared spectra of remarkably high quality to be measured to below $10 \mathrm{~cm}^{-1}$. Fortunately, because the tolerances required for interferometry are proportional to the wavelength, it was a relatively simple matter to make interferometers for far-infrared measurements where the wavelengths could be as long as $1 \mathrm{~mm}$. The first commercial far-infrared Fourier transform spectrometers were based on the interferometers developed at the NPL. These instruments were made by Research and Industrial Instruments Co. (Strathclyde, UK) and 
Grubb-Parsons (Newcastle-upon-Tyne, UK). Research and Industrial Instruments Co. (RIIC) was taken over by Beckman in the early 1970s.

The first of these far-infrared interferometers, the RIIC FS-520, was introduced in 1964. As with most far-infrared spectrometers, the entire optical path was evacuated. The FS-520 incorporated a continuous drive, where the optical path difference could be varied at a rate between about 0.5 to $500 \mu \mathrm{m} \mathrm{s}^{-1}$, with $5 \mu \mathrm{m} \mathrm{s}^{-1}$ being a typical value for measurements between 100 and $400 \mathrm{~cm}^{-1}$. At this slow speed, the Fourier frequencies were well below $0.1 \mathrm{~Hz}$ so that the radiation from the mercury lamp source had to be modulated by a $13-\mathrm{Hz}$ chopper. The throughput allowed for this spectral region was very large and the FS-520 had a polyethylene terephthalate (PET) beamsplitter that was over $20 \mathrm{~cm}$ in diameter. (The most common commercial sources of PET are du Pont, who market it as Mylar $^{(}$, and ICI, who marketed it as Melinex ${ }^{\circledR}$. Beamsplitters fabricated from PET are commonly known in the USA as Mylar beamsplitters and will be referred to as such throughout the rest of this article.) After the beam passed through the sample compartment, the interference record was measured with a Golay detector, and the detector signal was demodulated with a lock-in amplifier. The instrument was equipped with a Moiré fringe reference device that actuated a punched paper tape at equal intervals of path difference and the spectrum was computed off-line. As crude as this instrument sounds, its sensitivity was remarkably high, in part because of its very high optical throughput.

By about 1965, the NPL group had switched to a much more compact and easily fabricated design that became the basis of the Grubb-Parsons Mark II interferometer, a photograph of which can be seen in the first article of this volume. This interferometer, 
which was an almost exact replica of the NPL "cube", operated in the step-and-integrate (step-scan) mode in which the moving mirror was driven by a precise stepping motor so that there was no need for any fringe referencing. It allowed spectra to be measured at resolutions up to $\sim 0.1 \mathrm{~cm}^{-1}$. It may be noted that efficient vibration isolation was needed to obtain the best performance of this instrument.

These two instruments were so successful that they spawned a number of other slow-scan or step-scan interferometers for far-infrared spectrometry made by BeckmanRIIC in the UK, Coderg in France, and Polytec in Germany. By the mid-1970s, however, they started to be superseded by rapid-scanning interferometers that had started to gain popularity for mid-infrared measurements. Since these instruments are identical to most rapid-scanning interferometers used for mid-infrared spectrometry, and these instruments were described in the article in this Handbook by Jackson [3], we will not consider their design here.

\subsection{Beamsplitters for Far-Infrared Interferometers}

The biggest drawback to the use of Michelson interferometers for far-infrared spectrometry was the low efficiency of Mylar beamsplitter. The modulation efficiency of the interferometer, $\eta(\tilde{v})$, is given by $4 \boldsymbol{R}_{0}(\tilde{v}) \boldsymbol{T}_{0}(\tilde{v})$, where $\boldsymbol{R}_{0}(\tilde{v})$ and $\boldsymbol{T}_{0}(\tilde{v})$, are the reflectance and transmittance of the beamsplitter at wavenumber $\tilde{v}$, respectively. The maximum efficiency is found when $\boldsymbol{R}_{0}(\tilde{v})=\boldsymbol{T}_{0}(\tilde{v}),=0.5$, in which case $\eta(\tilde{v})=1$.

The sinusoidal variation of modulation efficiency with wavenumber is known variously as "interference fringes", "channel spectra", "dielectric resonances" and 
"Fabry-Perot fringes". If one considers the waves on a non-absorbing, parallel-sided sheet of a dielectric , the reflectance and transmittance are:

$$
R_{0}=\frac{2 R^{2}(1-\cos \delta)}{1+R^{2}-2 R \cos \delta}
$$

and

$$
T_{0}=\frac{(1-R)^{2}}{1+R^{2}-2 R \cos \delta}
$$

where $\delta=4 \pi \tilde{v} n_{t} d \cos \theta_{t}$ is the relative phase shift (in radians) between two adjacent emerging rays, $d$ is the thickness of the film, $n_{t}$ is its refractive index, and $\theta_{t}$ is the angle of the beam inside the film to the surface normal. The beamsplitter efficiency has maxima at $\delta=(2 m-1) \pi$ and minima at $\delta=2 m \pi$, for $m=1,2,3, \ldots$

Radiation polarized with its electric field polarized parallel to the plane of incidence is denoted by $p$, while radiation polarized with its electric field polarized perpendicular to the plane of incidence is denoted by $s$. For any non-zero angle of incidence at the beamsplitter, the reflectance for $p$ - and $s$ - polarized radiation have different forms.

$$
R_{p}=\frac{\tan ^{2}\left(\theta_{i}-\theta_{t}\right)}{\tan ^{2}\left(\theta_{i}+\theta_{t}\right)}
$$

and

$$
R_{s}=\frac{\sin ^{2}\left(\theta_{i}-\theta_{t}\right)}{\sin ^{2}\left(\theta_{i}+\theta_{t}\right)}
$$

where $\theta_{i}$ and $\theta_{t}$ are the angles of incidence and transmission, respectively, and are related by $n=\sin \theta_{t} / \sin \theta_{t}$. In a conventional Michelson interferometer, $\theta_{i}=45^{\circ}$. The refractive index of PET is about 1.6 and the index of absorption $k=0$ at most wavenumbers in the 
far infared. This yields $R_{p}=0.013$ and $R_{s}=0.115$. At a maximum in the beamsplitter response:

$$
T_{0}=\left(\frac{1-R}{1+R}\right)^{2}
$$

The relative beamsplitter efficiencies of a PET beamsplitter for the two polarizations are summarized in Table 1 . It can be seen that, because of the large difference in efficiencies for the $p$ and $s$ polarizations, a Michelson interferometer equipped with a PET beamsplitter is far more effective for $s$-polarized than $p$-polarized radiation.

Table 1: The beamsplitter efficiencies for $p$ and $s$ polarizations for a PET beamsplitter $\left(n_{t}\right.$ $=1.6)$ for a conventional $45^{\circ}$ Michelson interferometer

\begin{tabular}{|c|l|l|l|}
\hline Polarization & $R_{0}$ & $T_{0}$ & $4 R_{0} T_{0}$ \\
\hline$P$ & 0.052 & 0.95 & 0.20 \\
\hline$S$ & 0.37 & 0.63 & 0.93 \\
\hline None & 0.29 & 0.71 & 0.83 \\
\hline
\end{tabular}

Figure 3 shows the variation in the calculated efficiency of four common Mylar beamsplitters as a function of wavenumber for $s$-polarized radiation. To cover the entire far-infrared region from 400 to $10 \mathrm{~cm}^{-1}$ with a beamsplitter efficiency greater than 0.5 , it can be seen that several beamsplitters must be used. The spectral range typically covered 
by Mylar beamsplitters is shown in Table 2 . At long wavelengths $(>200 \mu \mathrm{m})$, where the efficiency of these beamsplitters is poor and black-body sources are weak, measurements become quite difficult. Measurement of a far infrared spectrum over the full range from 400 to $10 \mathrm{~cm}^{-1}$ using Mylar beamsplitters requires five different beamsplitters, as shown in Table 2.

Table 2: Spectral range covered by Mylar beamsplitters of different thicknesses

$\begin{array}{ll}\text { Thickness } & \text { Spectral Range } \\ 3 \mu \mathrm{m} & 450-120 \mathrm{~cm}^{-1} \\ 6 \mu \mathrm{m} & 450-120 \mathrm{~cm}^{-1} \\ 12.5 \mu \mathrm{m} & 225-60 \mathrm{~cm}^{-1} \\ 25 \mu \mathrm{m} & 100-30 \mathrm{~cm}^{-1} \\ 50 \mu \mathrm{m} & 50-15 \mathrm{~cm}^{-1} \\ 100 \mu \mathrm{m} & 25-8 \mathrm{~cm}^{-1} .\end{array}$

The beamsplitter efficiency may be improved if a material with a larger refractive index than Mylar is used. The data in Figure 4 show the variation of $R_{0}$ and $T_{0}$ for both $s$ - and $p$-polarized radiation as a function of the refractive index of the material from which the beamsplitter is fabricated for incidence angles of $45^{\circ}$ and $15^{\circ}$. The difference between the values of $R_{0}$ for $s$ and $p$ polarization ( $\mathrm{R}_{0 \mathrm{~s}}$ and $\mathrm{R}_{0 \mathrm{p}}$, respectively) may be reduced not only by choosing a material with higher refractive index such as silicon ( $n \sim$ 3.4) or germanium ( $n \sim 4.0)$, but also by reducing the angle of incidence at the 
beamsplitter. The values of $R_{0 s}$ and $R_{0 p}$ are much more similar at $15^{\circ}$ incidence, but to design an interferometer for which the incidence angle is so small is not trivial.

Several manufacturers of FT-IR spectrometers have applied variants of this technology in their far-infrared spectrometers. For example, Nicolet Instrument Corporation (Madison, WI, USA) [4] have used solid silicon plates for their far infrared beamsplitters. (Thick Ge plates cannot be used as far-infrared beamsplitters because of the strong phonon absorption of germanium.) If the Si plate is several millimeters thick, then the beamsplitter response consists of many closely spaced cycles (spacing $\sim 0.7 \mathrm{~cm}^{-1}$ for a 2-mm piece of silicon) that gives a single-beam background spectrum that is quite unsuitable for spectroscopic applications. If the resolution required is less than this spacing, e.g., $\Delta \tilde{v} \leq \sim 2 \mathrm{~cm}^{-1}$, then only the average beamsplitter response of $4 R_{0} T_{0} \sim 0.73$ is observed over essentially the entire wavenumber range. This beamsplitter has excellent long-wavelength response and has been used to obtain spectral information down to $1 \mathrm{~cm}^{-1}$. Provided that the sides are highly parallel, this beamsplitter should operate continuously into the mid infrared, with the exception of a strong phonon band at $\sim 620 \mathrm{~cm}^{-1}$. Except in those cases where very high resolution is required, a thick $\mathrm{Si}$ beamsplitter can replace all of the Mylar beamsplitters, as demonstrated by the data in

\section{Figure 5.}

If high resolution is required, however, the Si beamsplitter should be only a few micrometers in thickness and it is difficult to produce a free-standing Si film with highly parallel surfaces that is so thin. To circumvent this problem, Bruker Optics (Karlsruhe, Germany) have deposited a thin film of germanium onto a PET substrate. The result is a multilayer dielectric germanium beamsplitter that has all the advantages of the thick 
beamsplitter discussed previously, but it can be used for measurements at higher resolution and, because of the very thin Ge coating, the absorption caused by phonons is minimal. The improved efficiency at long wavelength that is achieved after coating a 6$\mu \mathrm{m}$ Mylar beamsplitter with a thin film of germanium is seen by the data in Figure 6 . The inset in this figure is for the same source/detector combination, only with a filter cooled to $4.2 \mathrm{~K}$ with a $100-\mathrm{cm}^{-1}$ cut-off in place. Cutting off the high frequency region of the spectrum shows the advantage of the coated beamsplitter at low frequency even more effectively.

Using a similar rationale, Perkin-Elmer (Beaconsfield, UK) have successfully vapor-deposited thin irregular metal layers on a stretched polypropylene substrate for use as a beamsplitter with wide wavelength coverage. Both metal mesh and metal layers deposited in a "polka-dot" pattern on a dielectric substrate can make very efficient beamsplitters at long wavelengths. Metal layers deposited in a "polka-dot" pattern are essentially the inverse of a metal mesh. Either a metal mesh or the polka-dot require an optimized pattern for high efficiency.

\subsection{Rapid-Scanning Interferometers for Far-Infrared Spectrometry}

Even though excellent spectra were measured using the interferometers described in the previous section, the outstanding performance of laser-referenced rapid scanning interferometers for mid- and near-infrared spectrometry led instrument manufacturers to modify these instruments for far-infrared operation. At first, the only change made in the interferometer when switching from mid-infrared to far-infrared operation was the substitution of a Mylar beamsplitter for the $\mathrm{Ge} / \mathrm{KBr}$ beamsplitter. At first, however, the performance of these instruments was quite poor because of several effects. First, as 
noted in the previous section, the efficiency of the Mylar beamsplitter is poor. Secondly, the Mylar film (which is stretched taut but is still quite flexible) can be easily set into vibration when the moving mirror reaches the end of its scan and is rapidly decelerated, causing the interferogram to be extremely unreproducible. (This effect has been called “drum-heading".)

In principle, the optical bench of a far-infrared can be either evacuated or purged to eliminate water vapor. For many reasons, however, evacuated instruments are far preferable to purged spectrometers for far-infrared operation. For example, it is extremely difficult to remove all traces of water vapor from the single-beam spectrum and the effect of residual rotational lines of water vapor are commonly seen in ratioed spectra, especially when they are measured at high resolution Furthermore, in a purged system vibrations caused by the incoming gas can create a real problem, leading to artifacts in the spectrum. Even low-frequency vibrations caused by the effect of people walking nearby the instrument and building vibrations also led to interferences below 200 $\mathrm{cm}^{-1}$, so that careful vibration and electrical isolation is called for. Finally, another factor that degrades the performance of far-infrared spectrometers based on rapid-scanning interferometers is interference by harmonics of the line frequency ( $60 \mathrm{~Hz}$ in the USA, 50 $\mathrm{Hz}$ in the UK.) When the velocity of the moving mirror is set to give a laser frequency of $5 \mathrm{kHz}$ (the most common velocity for operation with a pyroelectric detector), the "glitch" caused by the $60-\mathrm{Hz}$ interference is at $\sim 190 \mathrm{~cm}^{-1}$, which is right in the middle of the farinfrared region.

Some of these problems were easily overcome. The turn-around of contemporary interferometers is far smoother and severe drum-heading is less commonly seen in 
contemporary interferometers. To a greater or lessser effect, however, drum-heading is still frequently seen even in contemporary interferometers. The effect of vibrations and electrical interference has been overcome by better mechanical and electronic design. Evacuated instruments were designed and built to preclude interference by water vapor. The performance was still rather poor, however, because of the low efficiency of PET beamsplitters. If measurements are only required down to $180 \mathrm{~cm}^{-1}$, beamsplitters made with an analogous optical design to mid-infrared beamsplitters can be used, with CsI. replacing the $\mathrm{KBr}$ substrate and compensator plate and a thicker layer of germanium being used. For measurements below $\sim 180 \mathrm{~cm}^{-1}$, however, either Mylar or some of the special beamsplitters described in the previous section, are required.

\subsection{The Genzel Interferometer}

Another innovative concept for far-infrared spectroscopy is being used by Bruker in their in their IFS113v spectrometer which incorporates a Genzel interferometer. The design of this instrument is shown in Figure 7. Unlike a standard Michelson interferometer where the light is collimated at the beamsplitter and is never focused until it emerges, in the Genzel interferometer the light is focused onto the beamsplitter. The beams that are transmitted and reflected by the beamsplitter pass to two collimating mirrors and hence to a double-sided moving mirror. As the mirror moves, the path in one arm increases and the path in the other arm decreases. The small size of the beamsplitter in the Genzel interferometer allows several beamsplitters to be mounted on a wheel and interchanged without breaking the vacuum. The reduction in size significantly reduces the amplitude of the drum-head vibrations commonly associated with large-diameter Mylar beamsplitters. Another feature of the Genzel interferometer is that the angle of 
incidence at the beamsplitter $\left(\sim 15^{\circ}\right)$ is much smaller than a standard Michelson interferometer. This has the advantage of reducing the difference between the $s$ and $p$ polarizations (as shown in Figure 4), allowing optical polarization studies without having to reorient the sample.

For a displacement, $x$, of the moving mirror, an optical path difference of $4 x$ is generated by the Genzel interferometer Although this has been claimed as an advantage for the Genzel over the Michelson interferometer, any tilt introduced on moving the mirror leads to a doubling of the error in comparison to the error introduced in a standard Michelson interferometer with the same tilt. Thus we do not consider this to be a major advantage. Nonetheless, the Genzel interferometer does have several significant advantages for far-infrared spectroscopy.

\subsection{Interferometers for Very High-Resolution Spectrometry in the Far Infrared}

To obtain very high resolution measurements, some form of tilt compensation is required. To date, the most successful type of interferometer for very high resolution farinfrared spectrometry is the cube-corner. The highest resolution measurements achieved with such an instrument were reported by Kauppinen et al. [5] using the interferometer shown schematically in Figure 8. The cube corner retroreflectors were fabricated from $15 \times 15 \mathrm{~cm}$ flat mirrors and the diameter of the beamsplitter was $31 \mathrm{~cm}$. The maximum optical path difference, $\Delta_{\max }$, obtained with this interferometer was 6.2 meters. Since the full-width at half height of a sinc instrument line shape function is $0.605 / \Delta_{\max }$, the resolution obtained with this instrument should be approximately $0.001 \mathrm{~cm}^{-1}$. Spectra could be measured at a resolution of $0.002 \mathrm{~cm}^{-1}$ at $666 \mathrm{~cm}^{-1}$ using a Golay detector. 
When equal or higher resolution at wavenumbers below $100 \mathrm{~cm}^{-1}$ was desired, a liquidhelium cooled bolometer was needed.

Bruker Optics now market a cube-corner interferometer based on similar principles to Kauppinen's interferometer. The IFS $120 \mathrm{HR}$ has a maximum optical retardation of greater than $7.1 \mathrm{~m}$, giving an achievable resolution of better than 0.001 $\mathrm{cm}^{-1}$. One line in the rotation spectrum of $\mathrm{CO}$ at $61.42 \mathrm{~cm}^{-1}$ is shown in Figure 9, demonstrating that the theoretical resolution is being achieved. To give an idea of the high information content of spectra measured on this instrument, the spectrum of $\mathrm{H}_{2} \mathrm{~S}_{2}$ over a wavenumber range of $0.75 \mathrm{~cm}^{-1}$ measured at a resolution of $0.0025 \mathrm{~cm}^{-1}$ with a mercury arc source and LHe cooled bolometer is shown in Figure 10.

With a $125 \mu \mathrm{m}$ PET beamsplitter and a liquid-helium cooled bolometer, the instrument can be used down to $5 \mathrm{~cm}^{-1}$ (although measurements at the limit of the wavelength range are not necessarily practical at the highest resolutions). A detailed description of the design of the interferometer used in this instrument can be found in the article in this Handbook by Jackson [3]. It is a Michelson type that utilizes cube-corner retroreflectors, and has a clear aperture of more than $6 \mathrm{~cm}$. To reduce polarization effects, and increase the effective area of the beamsplitter, the angle of incidence, $\theta_{i}$, is $30^{\circ}$. Because of the very long optical paths, to avoid problems with water vapor in high resolution measurements the instrument can be evacuated to $2 \times 10^{-3}$ Torr.

\subsection{The Lamellar Grating Interferometer}


Because of the low reflectance of Mylar beamsplitters with $p$-polarized radiation, the modulation efficiency of Mylar beamsplitters to unpolarized radiation never exceeds $50 \%$, even at their optimum wavelength. For measurements below $50 \mathrm{~cm}^{-1}$, where the source intensity is extremely low, such low efficiency cannot be tolerated. One way around this problem is to use a lamellar grating interferometer, for which a beamsplitter is not required.

A schematic of a lamellar grating interferometer is shown in Figure 11. Unlike the Michelson interferometer, which operates on the principle of amplitude division, lamellar grating interferometers operate on the principle of wavefront division. It consists of two sets of parallel interleaved mirrors, or facets. One set is fixed, while the . other set can move in a direction perpendicular to the plane of the fixed facets. When the moving facets have traveled a distance $d$, the path difference between the rays that strike the fixed facets and the rays that strike the moving facets is $2 d / \cos \alpha$, where $\alpha$ is the angle of incidence of the median ray of the beam. Because almost all the radiation from the source reaches the detector, the efficiency of a lamellar grating can be significantly higher than that of a Michelson interferometer with a PET beamsplitter.

The low-wavenumber cut-off $\tilde{v}_{\mathrm{L}}$ of a lamellar interferometer is determined by the cavity effect, by which the modulation of waves whose electric vector is parallel to the sides of the cavity starts to decrease. This occurs when $\tilde{v}_{\mathrm{L}}<(0.3 a)^{-1}$, where $a$ is the grating constant. Thus if the facets are $1 \mathrm{~cm}$ across, $\tilde{v}_{\mathrm{L}} \sim 3 \mathrm{~cm}^{-1}$. The lowest frequency that has been measured with a lamellar grating spectrometer is $1.5 \mathrm{~cm}^{-1}$. The highwavenumber limit results from the fact that the grating can be considered as a series of 
long rectangular slits. Diffraction of the beam at this grating results in the interference being cancelled at the exit aperture. The high- wavenumber limit $\tilde{\nu}_{\mathrm{H}}$ is given by $F / a S$, where $F$ is the focal length of the collimator and $s$ is the diameter of the exit aperture. In practice, $\widetilde{v}_{\mathrm{H}}$ rarely exceeds about $150 \mathrm{~cm}^{-1}$.

The lamellar grating interferometer is the instrument of choice for measurements made below about $20 \mathrm{~cm}^{-1}$, and Beckman-RIIC once sold one of these instruments (the FS-820) that was optimized for this spectral region. However, there now is little interest in the measurement of spectra at such long wavelengths, and there are no instruments of this type currently available commercially.

One other instrument that was developed in the late 1960s and early 1970 was the Martin-Puplett polarizing interferometer [6]. This instrument will be described in some detail by Polavarapu [7] and will not be described here. Suffice it to say that, like the lamellar grating spectrometer, the performance of the polarizing interferometer in the far infrared is also not limited by the poor efficiency of the PET beamsplitter and excellent results were obtained on this instrument.

\section{Window Materials for Far-Infrared Spectrometry}

Since $\mathrm{NaCl}$ and $\mathrm{KBr}$ are opaque below $\sim 650$ and $\sim 380 \mathrm{~cm}^{-1}$, respectively, they cannot be used as window materials for far-infrared spectroscopy. Cesium iodide is transparent to about $150 \mathrm{~cm}^{-1}$ and hence can be used, not only as a window for measurements down to this wavenumber, but also for the preparation of alkali halide disks. 
Silicon cuts on below $400 \mathrm{~cm}^{-1}$ and is often useful, although its high refractive index does lead to some loss of energy. Both quartz and sapphire also transmit at long wavelength. Diamond is transparent throughout the entire far infrared. However, its small size and high cost prevents it from being a general-purpose window. The main use of bulk diamonds for far-infrared spectrometry has been as a window of a Golay detector. On the other hand, thin diamond films may be fabricated by chemical vapor deposition have high strength and are not extortionately expensive.

The best general purpose window material for far-infrared spectroscopy is polyethylene. High-density polyethylene (HDPE) is often used as a window for gas and liquid cells; however, this material has a fairly weak crystal lattice mode at $72 \mathrm{~cm}^{-1}$. When windows are at least $5 \mathrm{~mm}$ thick, the transmittance of this band can be less than 50\%. Low-density polyethylene (LDPE) has lower crystallinity than HDPE, so that this band is far weaker in LDPE than HDPE. As a result, however, LDPE is also less rigid and not as suitable as HDPE as a window material. The melting point of LDPE is about $140^{\circ} \mathrm{C}$ and so this material is easily melted. One useful way of preparing solid samples for far-infrared spectroscopy is to grind them with powdered LDPE and compress the mixture while heating it to about $140^{\circ} \mathrm{C}$. In view of the fact that black-body sources emit much higher power in the mid- than the far-infrared region of the spectrum, it is often preferable to remove as much of the short-wavelength radiation as possible if very long wavelengths are to be measured. In this case, black polyethylene, of the type commonly used as garbage collection bags, comes in useful as an optical filter since it absorbs most of the energy above about $600 \mathrm{~cm}^{-1}$ while having a transmittance greater than $50 \%$ below $200 \mathrm{~cm}^{-1}$. 
Two other polyolefins that have been proposed for use as windows for farinfrared spectrometry are polypropylene (PP) and poly-(4-methyl-pentene-1), or TPX ${ }^{\circledR}$. These materials are structurally related as 4-methyl-pentene-1 is a propylene dimer. These materials have one great advantage over polyethylene for use as a window. Whereas polyethylene is typically barely translucent, PP and TPX are transparent to visible light. TPX is significantly more susceptible to air oxidation when illuminated with ultraviolet light, so that if a mercury lamp is used as the source, optical filtering, e.g., with black polyethylene, is necessary. Many far-infrared spectroscopists tend to use polypropylene thin films in preference to TPX when used in a vacuum, since PP has a fairly high burst pressure.

\section{Summary}

Fourier transform spectrometers developed in three distinct spectral regions in the early 1960s. Pierre Connes and his coworkers in France developed remarkably sophisticated step-scan interferometers that permitted near-infrared spectra to be measured with a resolution of better than $0.01 \mathrm{~cm}^{-1}$. These instruments may be considered the forerunners of the step-scan interferometers made by Bruker, Bio-Rad (Cambridge, MA, USA) and Nicolet although their principal application was in the field of astronomy. Low-resolution rapid-scanning interferometers were developed by Larry Mertz and his colleagues at Block Engineering (Cambridge, MA, USA) for remote sensing.

Nonetheless, the FT-IR spectrometers that are so prevalent in chemical laboratories today are direct descendents of these instruments. The interferometers that were developed for far-infrared spectrometry in Gebbie's laboratory have had no commercial counterparts for at least 15 years. However, it could be argued that these instruments did as much to 
demonstrate the power of Fourier transform spectroscopy to the chemical community as any of the instruments developed for mid- and near-infrared spectrometry. Their performance was every bit as good as today's rapid-scanning interferometers. However, the market for these instruments is so small today that it has proved more lucrative to modify rapid-scanning interferometers that were originally designed for mid-infrared spectrometry than to compete with these instruments with slow continuous scan or stepscan interferometers.

This work is supported, in part, by the United States Department of Energy, Division of Materials Science, under Contract No. DE-AC01-98CH10886.

\section{References}

1. G. P. Williams, "Synchrotron and Free Electron Laser Sources of Infrared Radiation", Handbook of Vibrational Spectroscopy, Volume 1, John Wiley and Sons, Chichester, UK (2001).

2. P. R. Griffiths and J. A. de Haseth, Fourier Transform Infrared Spectrometry, Wiley Interscience, New York, pp. 209-212 (1986).

3. R. S. Jackson, "Continuous-Scanning Interferometers for Mid-Infrared Spectrometry", Handbook of Vibrational Spectroscopy, Volume 1, John Wiley and Sons, Chichester, UK (2001).

4. Nicolet Instrument Corporation, U.S. Patent 4632553.

5. J. Kauppinen and V-M. Horneman, Appl. Opt., 30, 2575 (1991).

6. D. H. Martin and E. Puplett, Infrared Phys., 10, 105 (1969). 
7. P. Polavarapu, "Polarization Interferometers for Infrared Spectrometry",

Handbook of Vibrational Spectroscopy, Volume 1, John Wiley and Sons, Chichester, UK (2001). 


\section{Figure Captions}

1. Front section of a Golay detector. Chopped incident radiation passes through the window onto a blackened film, causing the pressure of the gas in the pneumatic chamber to fluctuate and a flexible mirror to deform with the same modulation frequency as the chopper. A pinhole leak minimizes the effect of long-term temperature variation. (Reproduced by permission of Cathodeon Ltd.)

2. Rear section of a Golay detector. Visible radiation is passed through a grating and is focused onto the flexible mirror shown in Fig. 1. The reflected radiation passes back through the grating and is measured by a light sensor. When the flexible mirror is undistorted, the grating prevents any light from reaching the detector. The greater the distortion of the mirror, the greater is the measured signal. (Reproduced by permission of Cathodeon Ltd.).

3. Variation in the efficiency of 3- $\mu \mathrm{m}, 6-\mu \mathrm{m}, 12-\mu \mathrm{m}$ and $25-\mu \mathrm{m}$ Mylar beamsplitters as a function of wavenumber for $s$-polarized radiation. .

4. Variation of $\mathrm{R}_{0}$ for $s$ - and $p$-polarized radiation as a function of the refractive index of the material from which the beamsplitter is fabricated at $45^{\circ}$ and $15^{\circ}$ incidence. Note the much smaller difference between the values of $R_{0 s}$ and $R_{0 p}$ at an incidence angle of $15^{\circ}$ compared to $45^{\circ}$.

5. Comparison of single-beam spectra measured with a $3-\mu \mathrm{m}$ and $12-\mu \mathrm{m}$ Mylar film and a 2-mm thick silicon beamsplitter. Note that the silicon beamsplitter covers a much wider spectral range than either of the Mylar beamsplittters. 
6. Single-beam spectra measured with an uncoated 6- $\mu \mathrm{m}$ Mylar beamsplitter and the same material coated with a thin layer of germanium. (Inset) The single-beam spectrum measured with the same source/detector combination, only with a filter cooled to $4.2 \mathrm{~K}$ with a $100-\mathrm{cm}^{-1}$ cut-off in place.

7. Genzel interferometer of the type incorporated in the Bruker IFS-113 FT-IR spectrometers. c, filter wheel, d, carousel on which up to six beamsplitters may be mounted, e. moving two-sided mirror, f. reference interferometer, g. He-Ne laser, h. spherical collimating mirror.

8. Optical layout of the high-resolution cube-corner interferometer reported by Kauppinen and Horneman. (Reproduced from reference [5] by permission of the Optical Society of America; copyright (C) 1991).

9. Line in the pure rotation spectrum of $\mathrm{CO}$ measured without apodization at the maximum resolution of the Bruker HR-002 spectrometer; the full-width at half height of this line is $0.00095 \mathrm{~cm}^{-1}$.

10. Far-infrared spectrum of $\mathrm{H}_{2} \mathrm{~S}_{2}$ over a wavenumber range of $0.75 \mathrm{~cm}^{-1}$ measured at a resolution of $0.0025 \mathrm{~cm}^{-1}$.

11. Principle of the lamellar grating interferometer. Radiation reflected off the moving facets travels a distance of $2 d / \cos \alpha$ further than the radiation reflected off the stationary facets. 


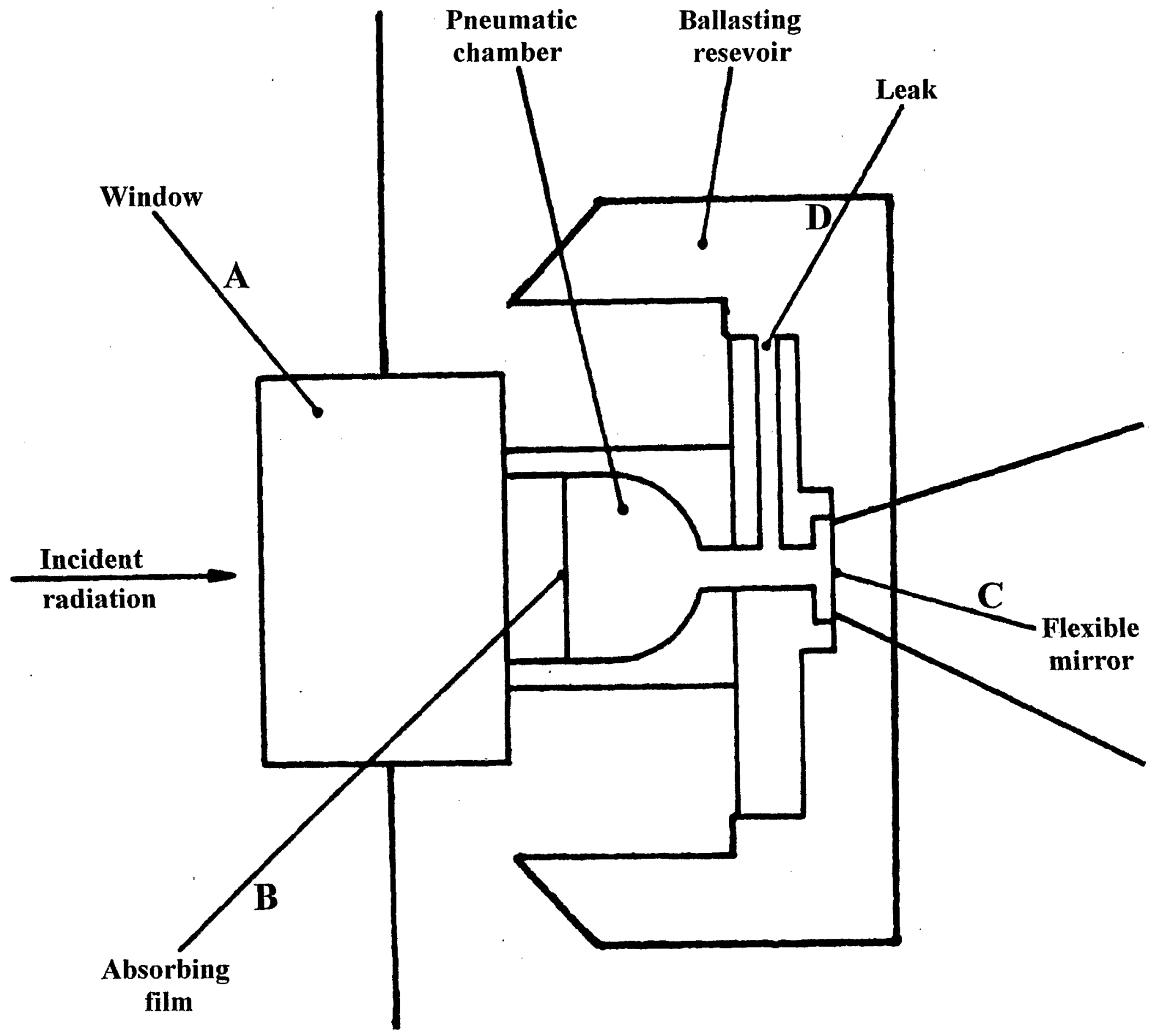




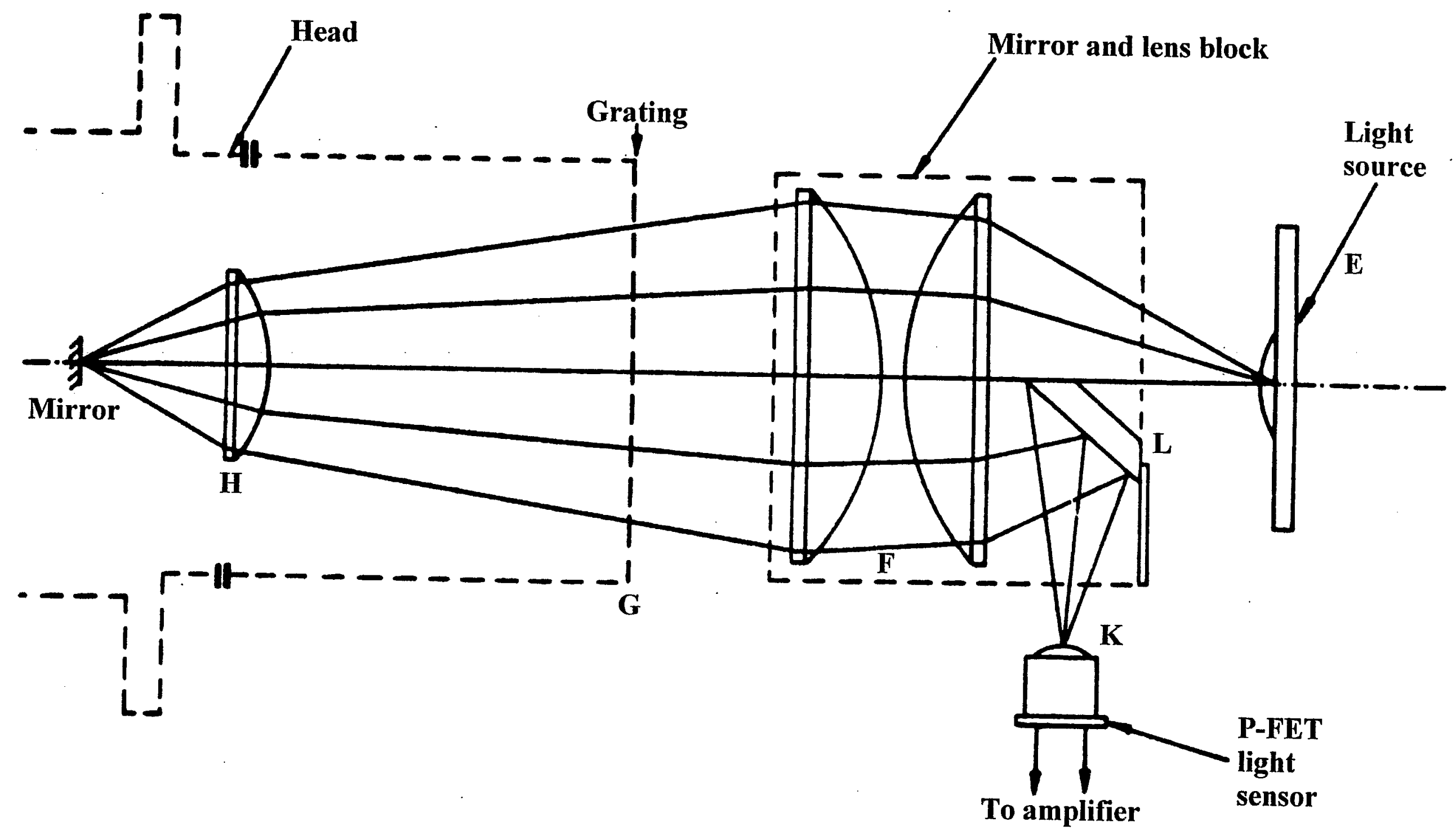




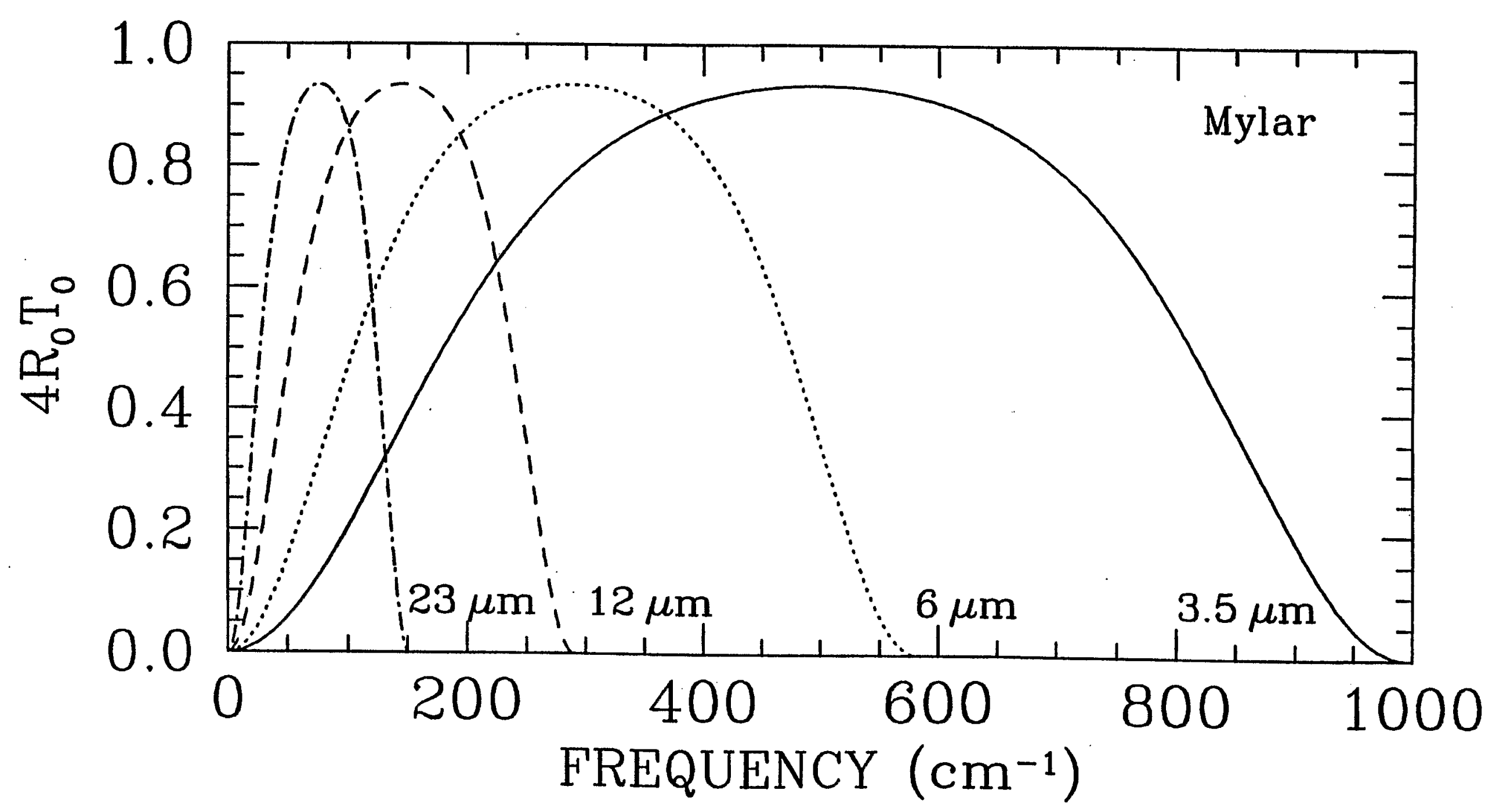




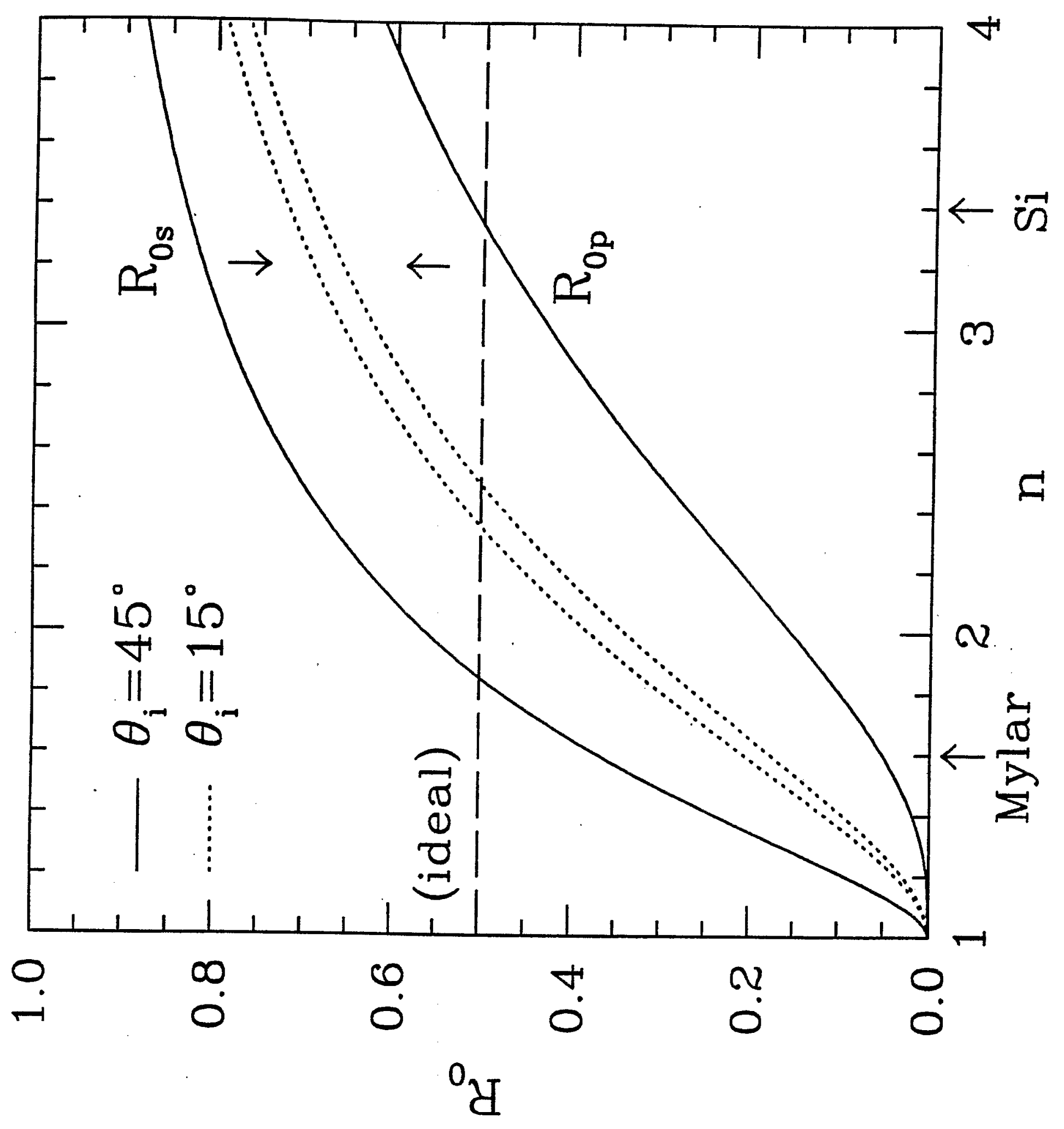




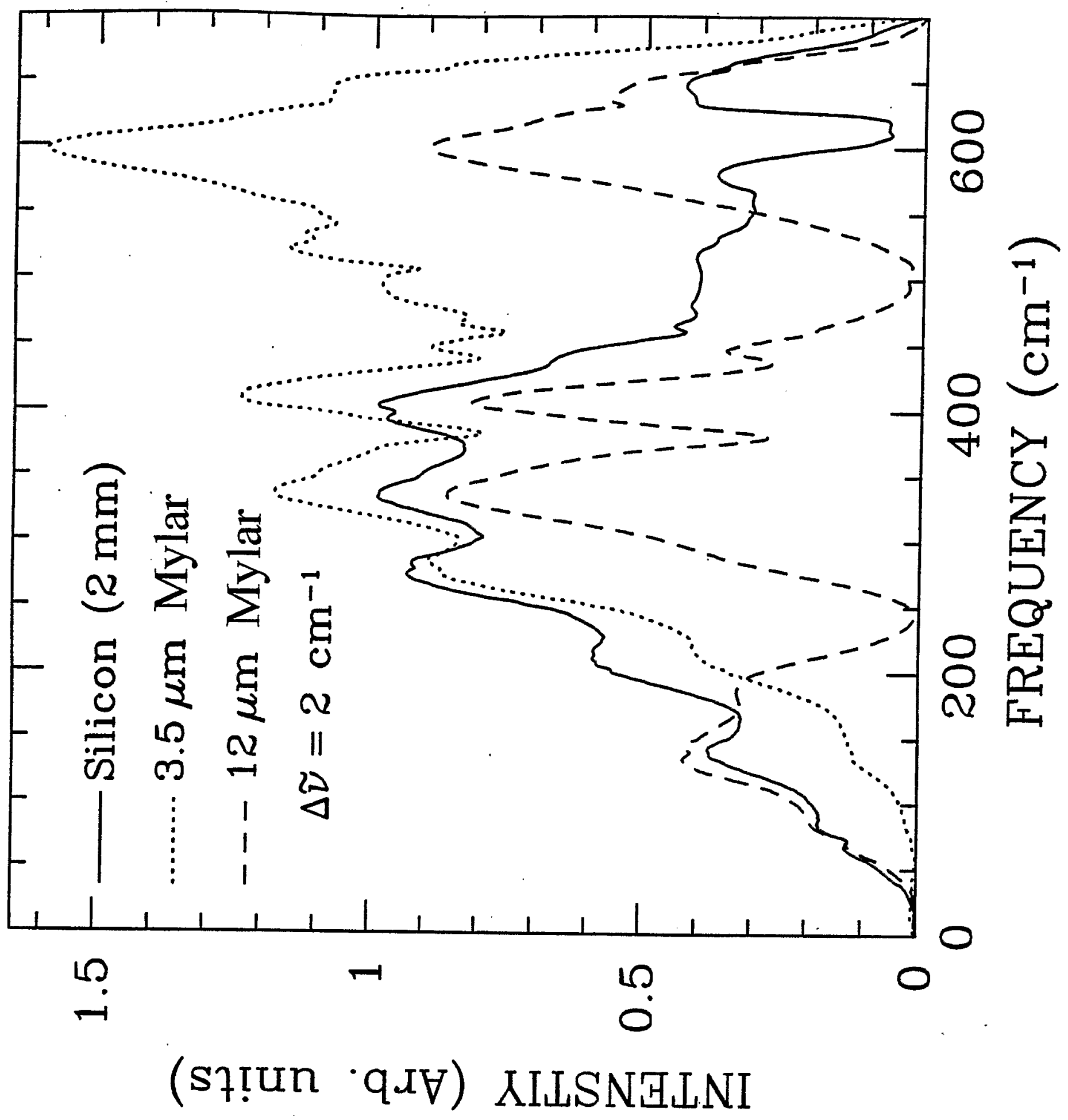




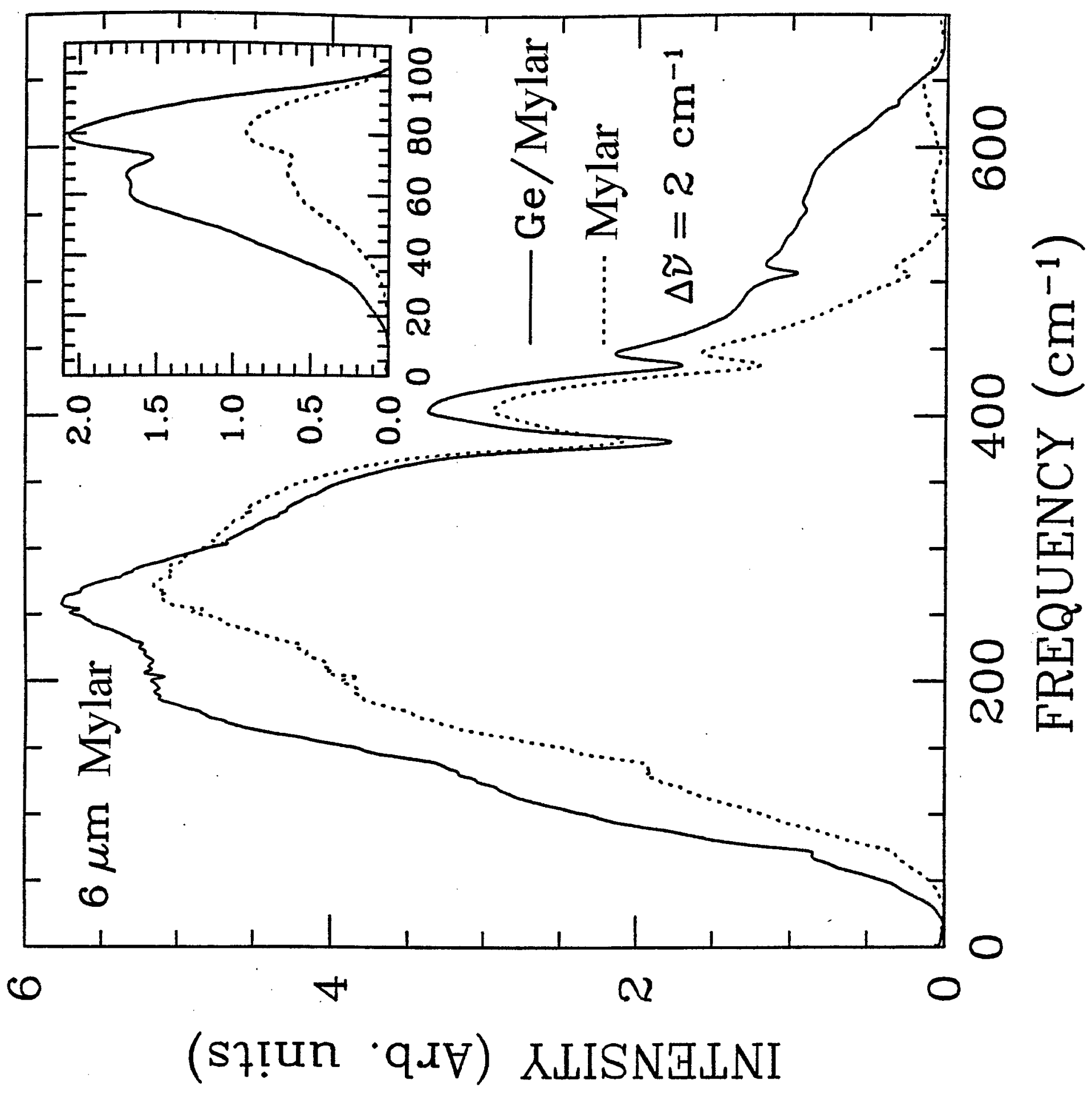




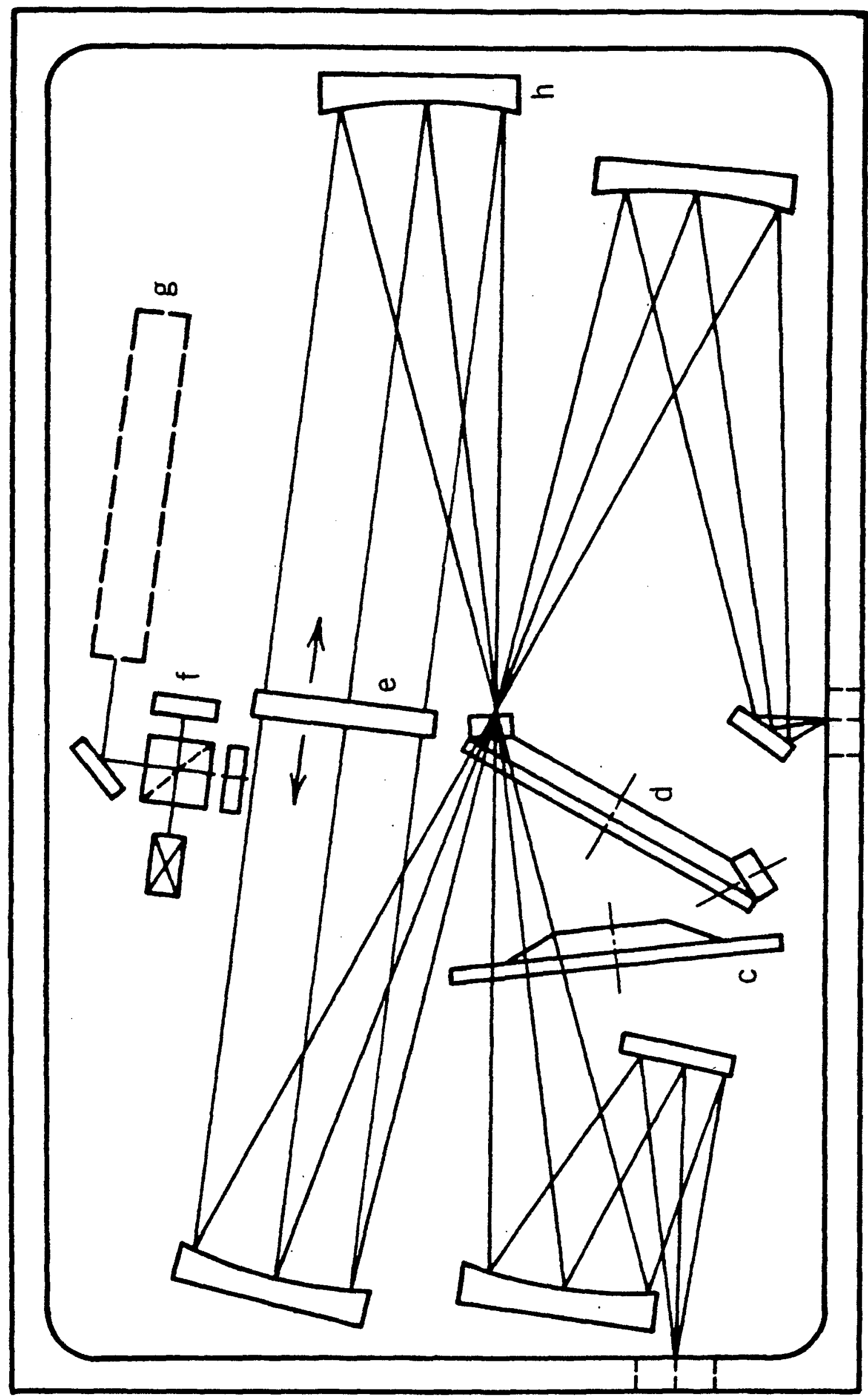




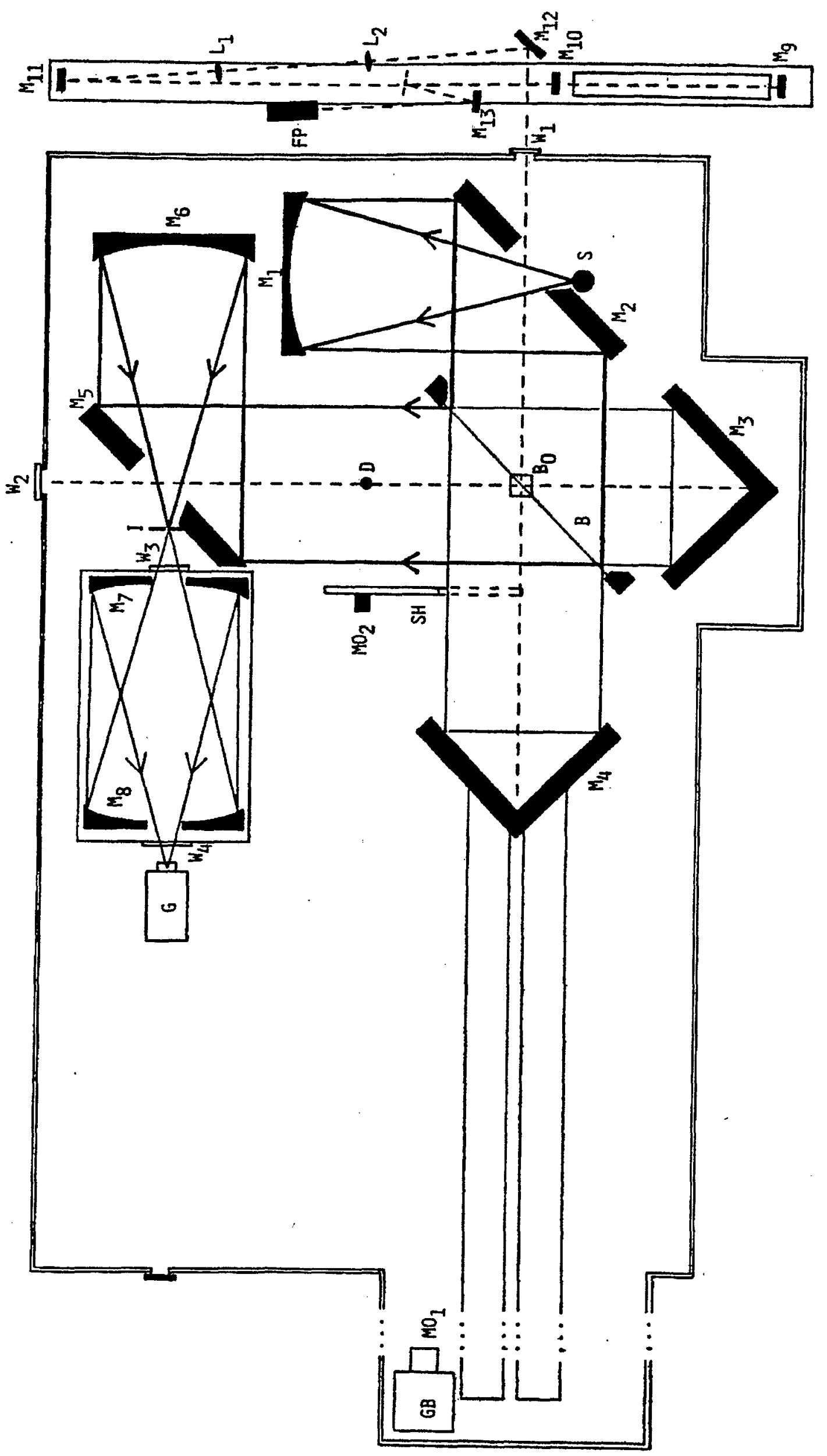




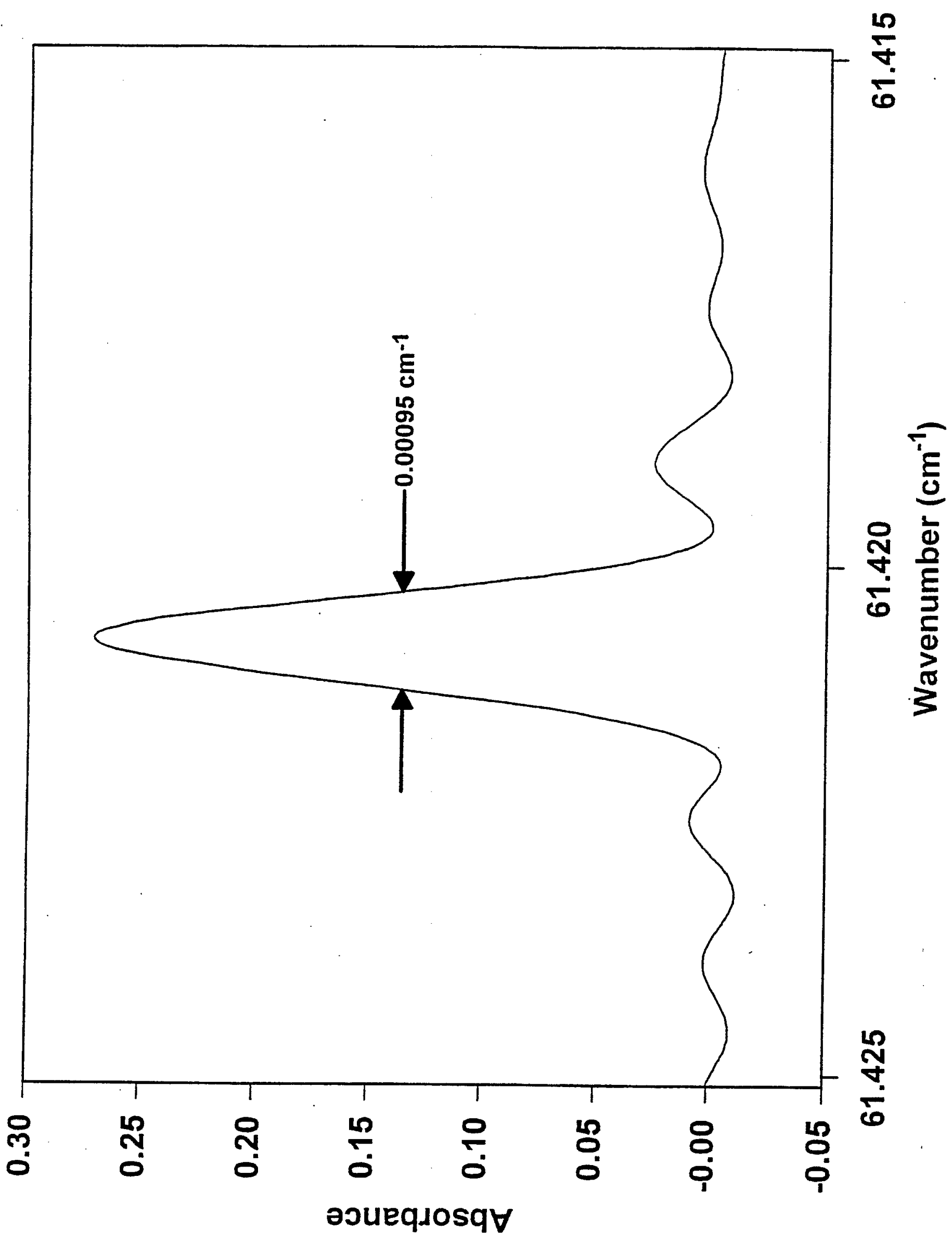




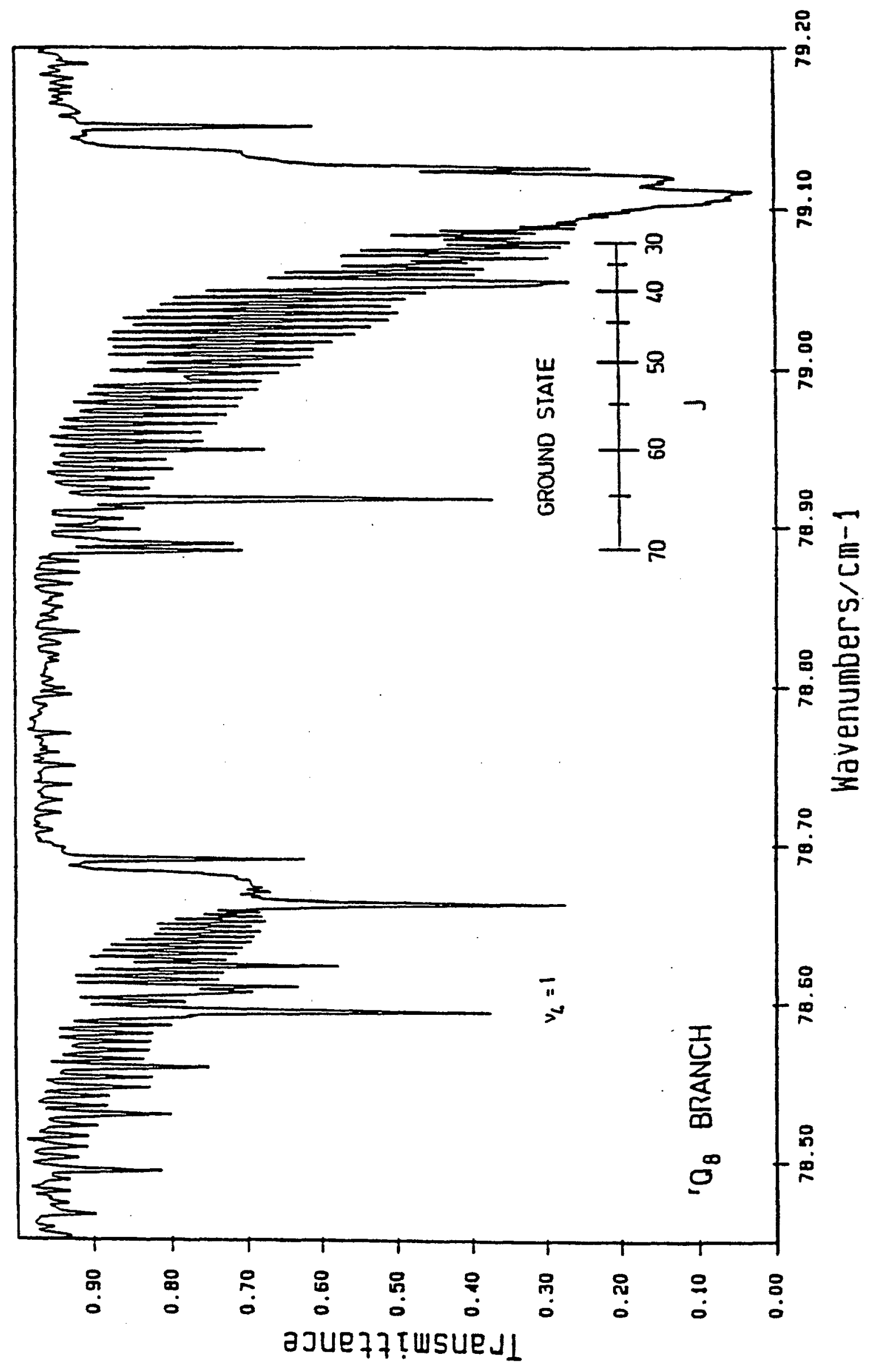



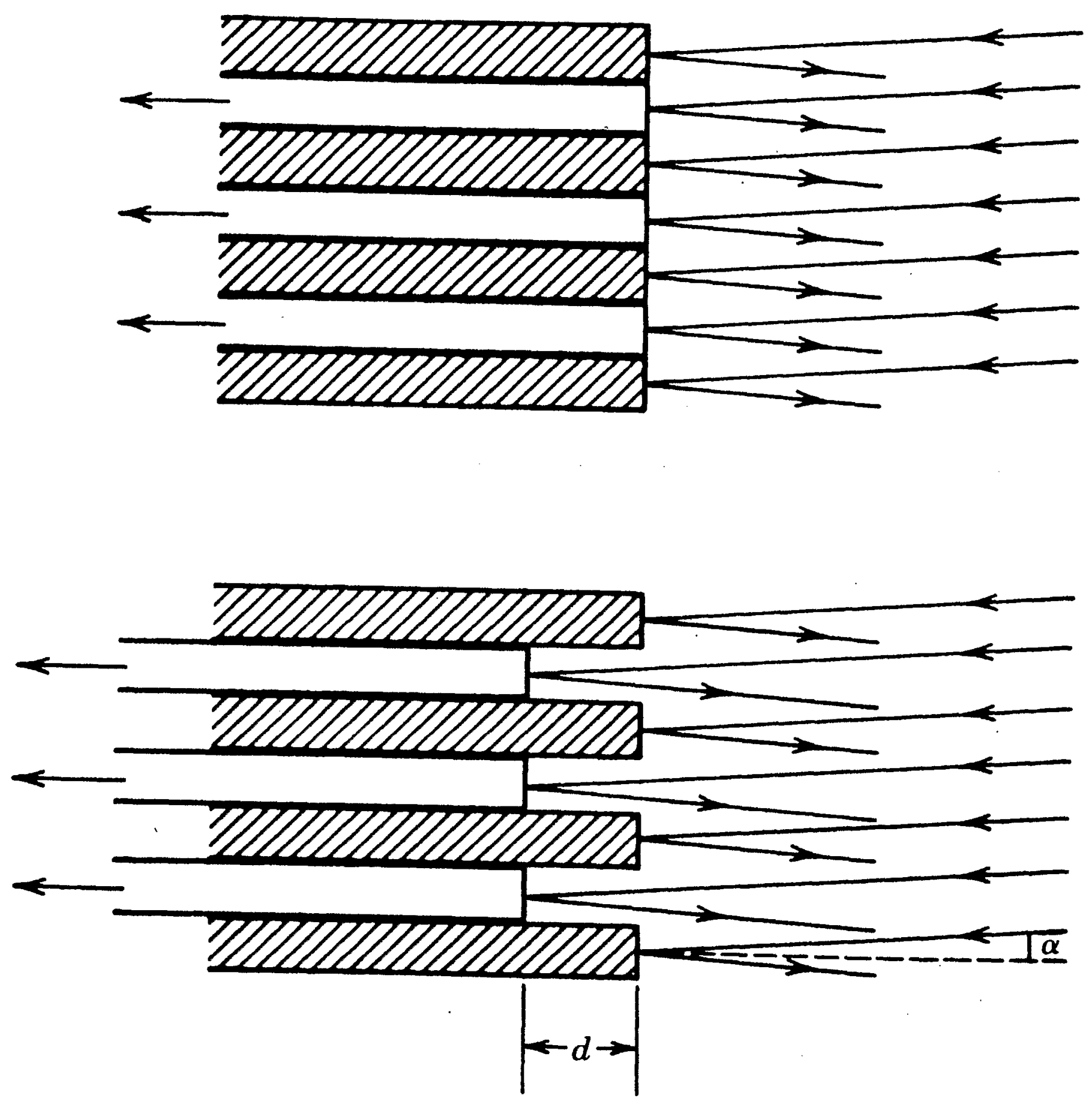


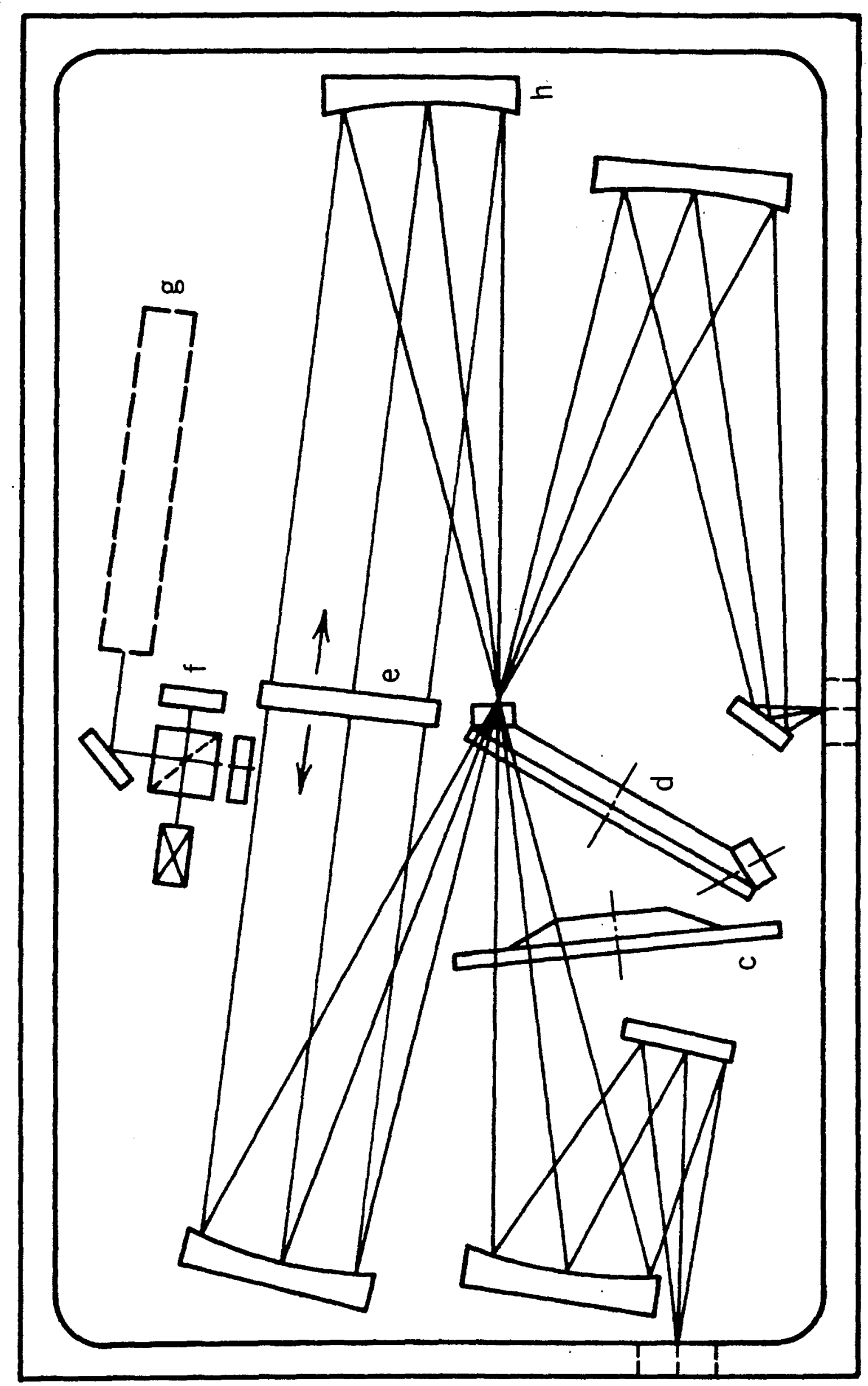




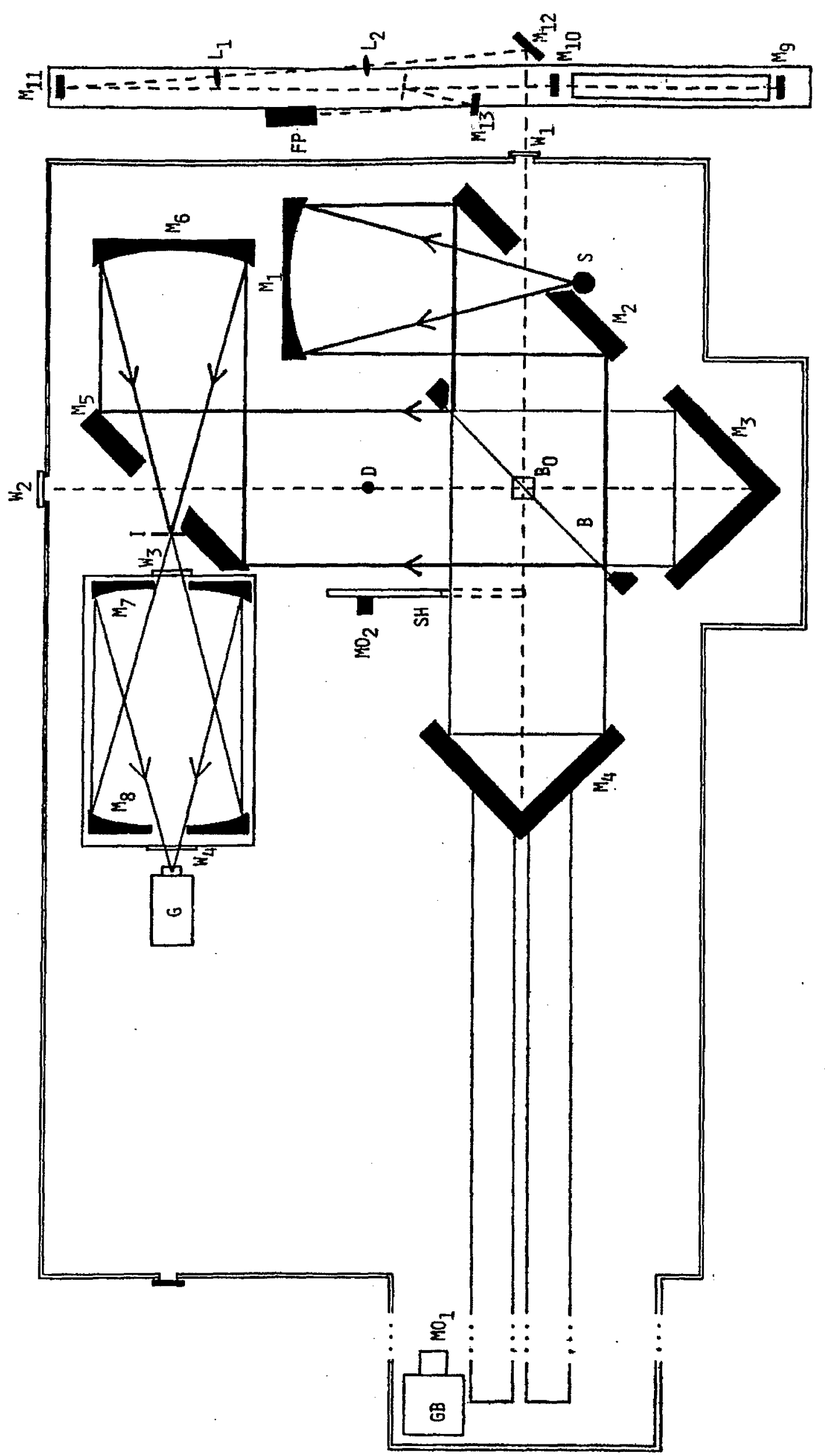




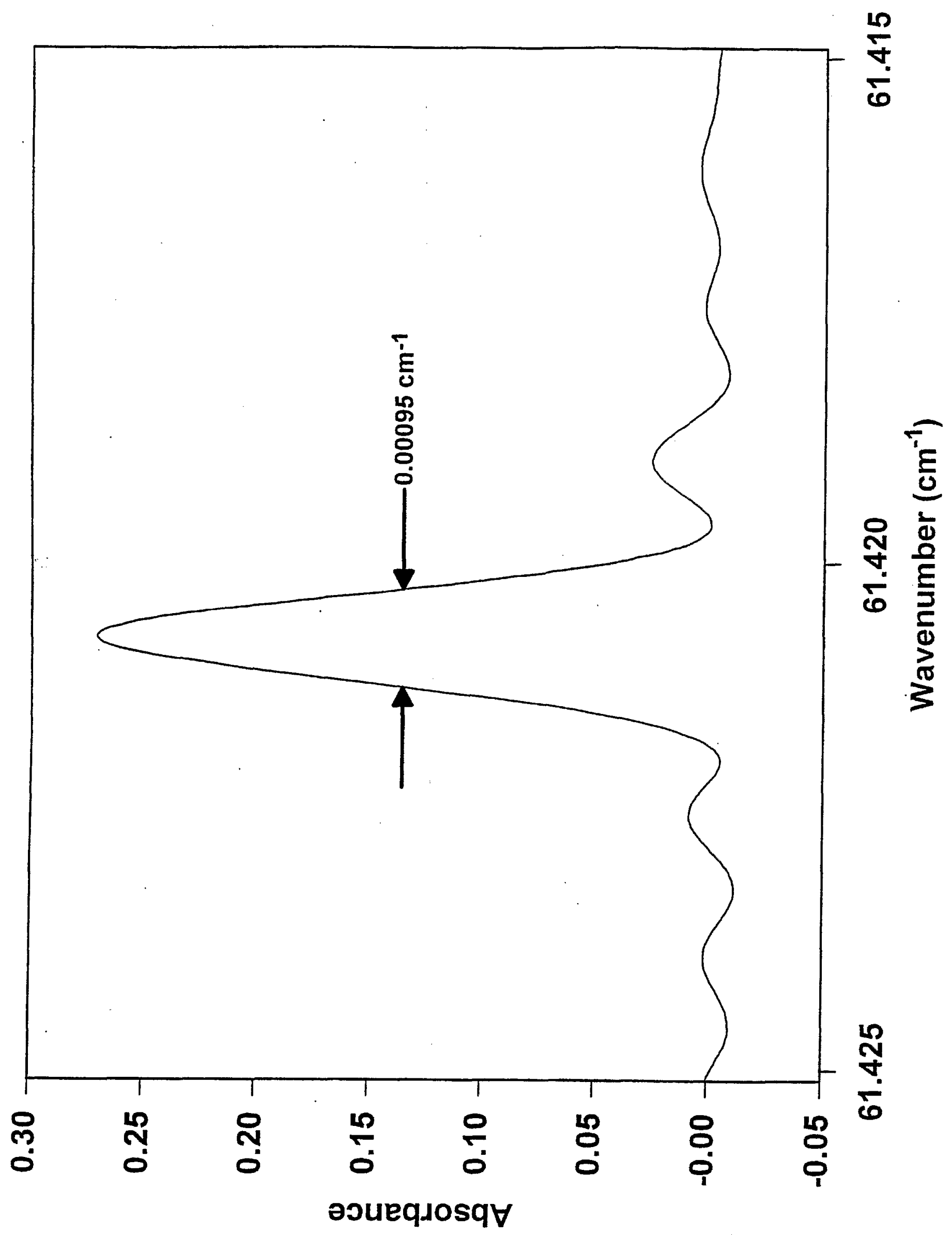




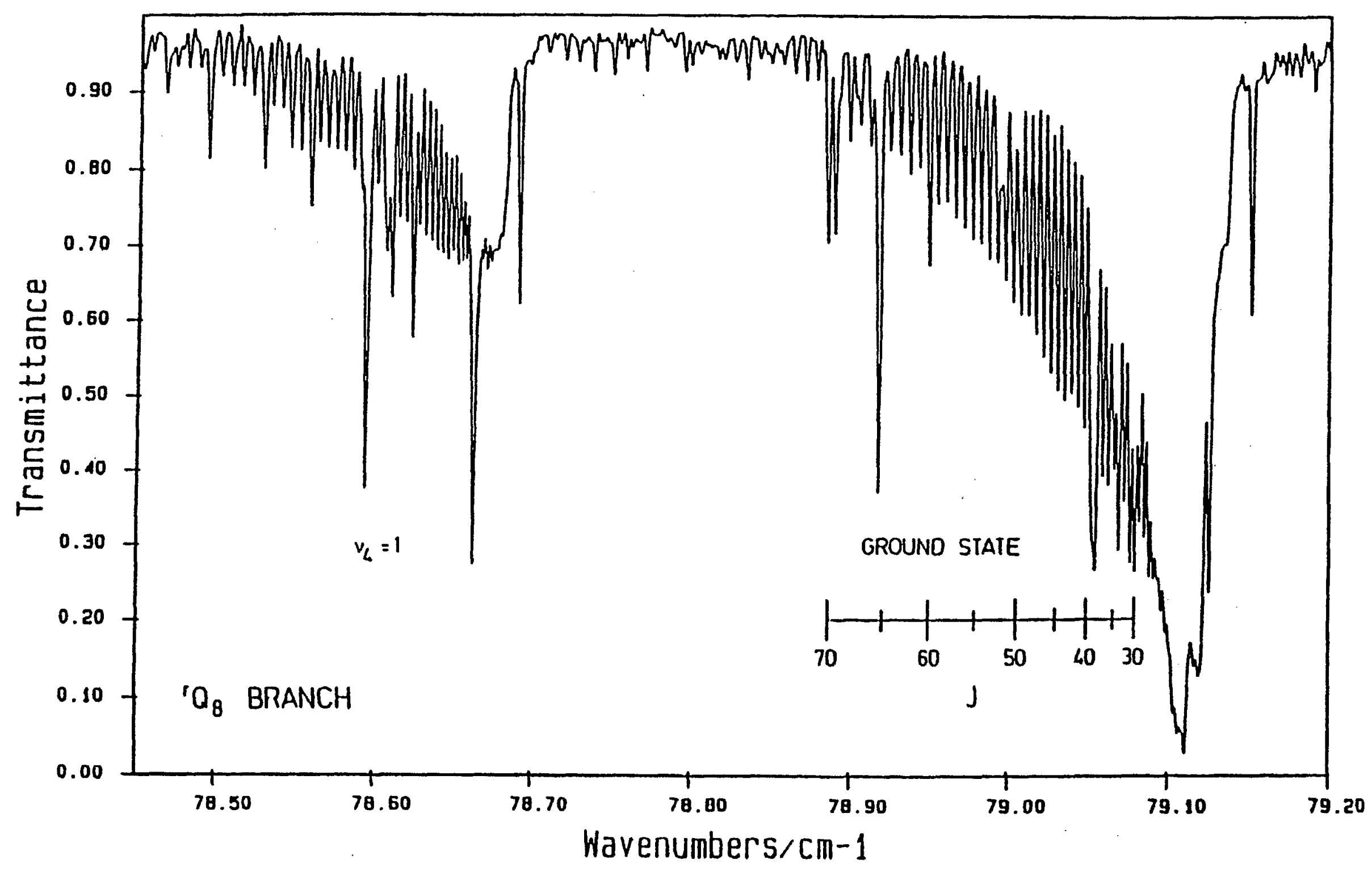



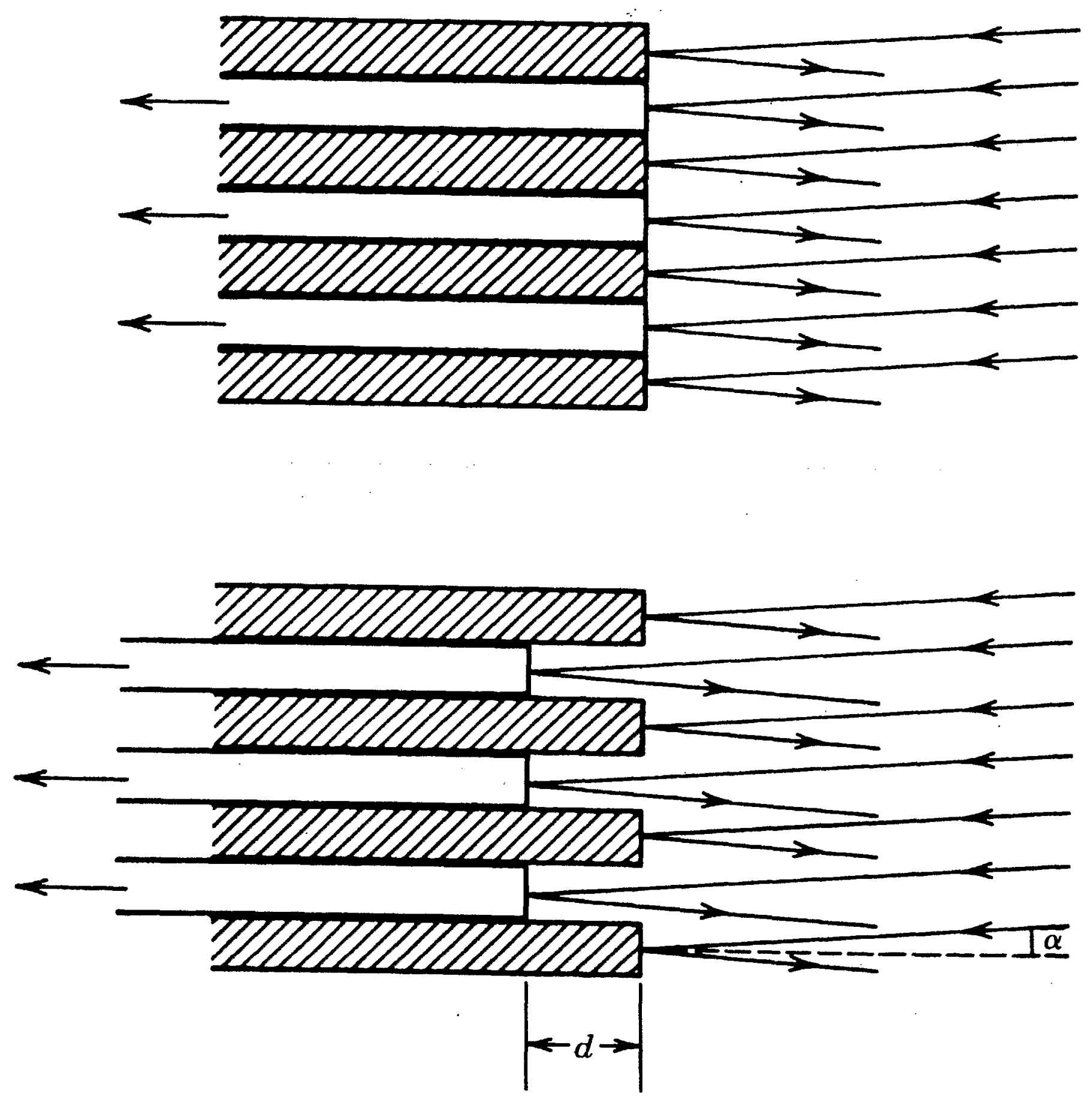